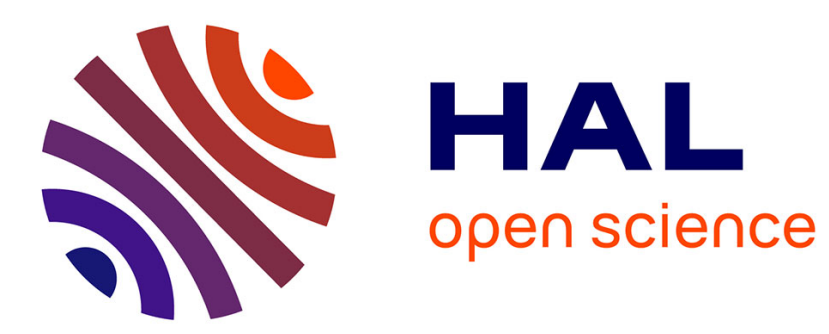

\title{
Why do larval helminths avoid the gut of intermediate hosts?
}

\author{
G.A. Parker, M.A. Ball, J.C. Chubb
}

\section{To cite this version:}

G.A. Parker, M.A. Ball, J.C. Chubb. Why do larval helminths avoid the gut of intermediate hosts?. Journal of Theoretical Biology, 2009, 260 (3), pp.460. 10.1016/j.jtbi.2009.06.016 . hal-00554630

\author{
HAL Id: hal-00554630 \\ https://hal.science/hal-00554630
}

Submitted on 11 Jan 2011

HAL is a multi-disciplinary open access archive for the deposit and dissemination of scientific research documents, whether they are published or not. The documents may come from teaching and research institutions in France or abroad, or from public or private research centers.
L'archive ouverte pluridisciplinaire HAL, est destinée au dépôt et à la diffusion de documents scientifiques de niveau recherche, publiés ou non, émanant des établissements d'enseignement et de recherche français ou étrangers, des laboratoires publics ou privés. 


\section{Author's Accepted Manuscript}

Why do larval helminths avoid the gut of intermediate hosts?

G.A. Parker, M.A. Ball, J.C. Chubb

PII:

S0022-5193(09)00281-1

DOI: doi:10.1016/j.jtbi.2009.06.016

Reference: YJTBI 5605

To appear in: $\quad$ Journal of Theoretical Biology

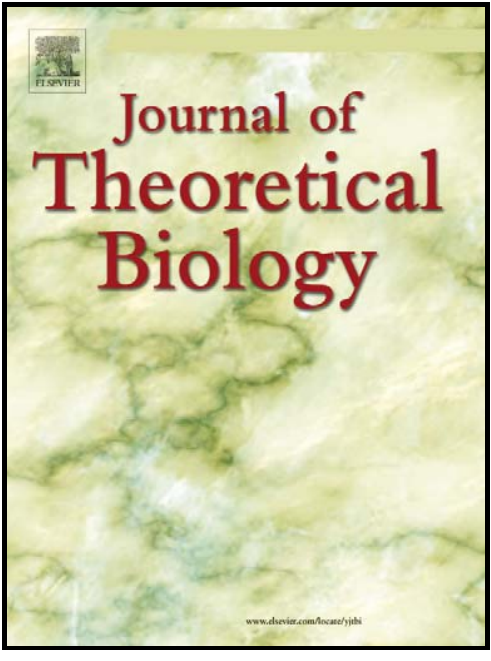

www.elsevier.com/locate/yjtbi

Received date: $\quad 12$ May 2009

Revised date: $\quad 16$ June 2009

Accepted date: $\quad 16$ June 2009

Cite this article as: G.A. Parker, M.A. Ball and J.C. Chubb, Why do larval helminths avoid the gut of intermediate hosts?, Journal of Theoretical Biology, doi:10.1016/j.jtbi.2009.06.016

This is a PDF file of an unedited manuscript that has been accepted for publication. As a service to our customers we are providing this early version of the manuscript. The manuscript will undergo copyediting, typesetting, and review of the resulting galley proof before it is published in its final citable form. Please note that during the production process errors may be discovered which could affect the content, and all legal disclaimers that apply to the journal pertain. 


\title{
Why do larval helminths avoid the gut of
}

\section{intermediate hosts?}

\author{
G. A. Parker ${ }^{1}$, M. A. Ball ${ }^{2}$ \& J. C. Chubb ${ }^{1}$ \\ ${ }^{1}$ Division of Population and Evolutionary Biology, School of Biological Sciences, University \\ of Liverpool, Liverpool L69 7ZB \\ ${ }^{2}$ Division of Applied Mathematics, Department of Mathematical Sciences, University of
}

Liverpool, Liverpool L69 3BX

\begin{abstract}
In complex life cycles, larval helminths typically migrate from the gut to exploit the tissues of their intermediate hosts. Yet the definitive host's gut is overwhelmingly the most favoured site for adult helminths to release eggs. Vertebrate nematodes with one-host cycles commonly migrate to a site in the host away from the gut before returning to the gut for reproduction; those with complex cycles occupy sites exclusively in the intermediate host's tissues or body spaces, and may or may not show tissue migration before (typically) returning to the gut in the definitive host. We develop models to explain the patterns of exploitation of different host sites, and in particular why larval helminths avoid the intermediate host's gut, and adult helminths favour it. Our models include the survival costs of migration between sites, and maximise fitness (= expected lifetime number of eggs produced by a given helminth propagule) in seeking the optimal strategy (host gut versus host tissue exploitation) under different growth, mortality, transmission and reproductive rates in the gut and tissues (i.e. sites away from the gut). We consider the relative merits of the gut and tissues, and conclude that (i) growth rates are likely to be higher in the tissues, (ii) mortality rates possibly higher in the gut (despite the immunological inertness of the gut lumen), and (iii) that there are very high benefits to egg release in the gut. The models show that these growth and mortality relativities would account for the common life history pattern of avoidance of the intermediate host's gut because the tissues offer a higher growth rate/mortality rate ratio (discounted by the costs of migration), and make a number of testable predictions. Though nematode larvae in paratenic hosts usually migrate to the tissues, unlike larvae in intermediates, they sometimes remain in the gut, which is predicted since mortality rate and migration costs alone determine the site to be exploited.
\end{abstract}




\section{Introduction}

Parasitic worms (helminths) have life cycles involving the exploitation of one or (typically) more hosts. Sexual maturation and reproduction occurs in the definitive host, and in most instances adults release propagules (usually eggs, but sometimes early stage larvae) to the environment. In one-host cycles, the propagules infect new definitive hosts, but more commonly they first infect an intermediate host (in which there is growth but no reproduction) before they transfer to the definitive host (two-host cycles) (Fig. 1). Some complex life cycles involve three (or occasionally more) hosts in succession: two intermediate hosts and finally a definitive host. The term 'intermediate host' is replaced with 'paratenic host' if there is no growth or development in that host. Transmission between hosts varies, but the commonest methods are trophic transmission (where the host ingests the parasite, either as a free-living propagule, or by predating upon an intermediate or paratenic host) or skin penetration (where the parasite invades the skin of the host).

One of the most pervasive features of trophically transmitted helminth complex life cycles is that larvae in their intermediate hosts penetrate the gut wall and migrate to some regular site in the tissues or body cavities, where they typically grow for some time until reaching a given size and stage of development, when growth ceases (termed growth arrest at larval maturity, or GALM; Ball et al. 2008, Parker et al. 2009). In contrast, in their definitive hosts (in complex or direct life cycles), while some species of nematodes (roundworms) migrate to the tissues and commonly return to the gut to release their eggs, almost all cestodes (tapeworms) and all acanthocephalans (spiny-headed worms) remain within the gut lumen to mature sexually and release eggs that are shed with the host faeces. Thus while tissue migration is ubiquitous in intermediate hosts, it is much less common in definitive hosts. Where transmission is by skin penetration as in many trematodes (flukes), larvae entering intermediate hosts also typically migrate to a site in the tissues or body spaces for growth. 
They depart from their intermediate host at an optimal body size (Iwasa \& Wada 2006), and many actively search for their next host. If this is the definitive host, the majority reside in the host gut for egg release (Chubb et al. submitted).

In the present paper we examine the prevalence of different patterns of host utilization across species of nematodes of vertebrates, and outline a prospective logic for the evolution of strategies of exploitation of the host's gut versus the host's tissues. We compare conditions for host tissue versus host gut exploitation in one-host cycles with two-host cycles (an outline of the two types of life cycle is given in Fig. 1). Though our models discriminate only between exploitation of the gut and the tissues, in reality there is great diversity in the sites that helminths exploit (see e.g. Crompton 1973, for the gut; Chubb et al. submitted). Sometimes only the gut is exploited (Fig. 1, pathway a); commonly a tissue migration occurs only as far as the gut mucosa (Fig. 1, pathway b); in other cases there are extensive migrations away from the gut to various host tissues or body spaces (Fig. 1, pathway c) such as the body cavity, the lungs, or the urinary bladder. We postulate that there are large differences in growth and survival rates between the gut and the tissues; an analysis of prospects in the gut compared to the tissues is therefore justifiable as a first step to understanding the multiplicity of helminth life histories. We build on the pioneering study of Read \& Skorping (1995) who showed that tissue migration of nematodes in definitive hosts might yield adaptive advantages in terms of accelerated growth. Our aims are to provide a life history strategy foundation for understanding the evolution of tissue migration, and particularly to explain why larval helminths avoid the gut of intermediate hosts. We recommend examination of our previous works on helminth life cycles (Parker et al. 2003, Ball et al. 2008, Parker et al. 2009), which the present analysis develops.

The complexity and diversity of helminth life cycles is so vast that exceptions to general predictions from prospective models are inevitable; we nevertheless hope that our analyses offer initial insights into an intriguing, yet under-investigated area. 


\section{The prevalence of various host exploitation strategies in nematodes}

Nematodes of vertebrates provide great diversity of life history patterns, ranging from one-host to three-host cycles, with considerable variation in both the occurrence and, when present, the form of tissue migration. In order to obtain an overall picture of the diversity in life history patterns of nematodes, we analysed the range of gut and tissue strategies in Anderson's (2000) treatise on the life cycles, using D to define a definitive host, I an intermediate host, and $\mathrm{P}$ a paratenic host (see Appendix A). The life history pattern in a given host was also defined; for example, $\mathrm{G}$ represents long-term residence in the gut, and $\mathrm{T}$ tissue migration (see Appendix A, Table A1a for full classification). The results (shown in Appendix A, Table A1b) are intended only to give a general impression of the relative occurrence of the various host exploitation strategies in nematodes of vertebrates, not as a detailed comparative survey. As measured by numbers of species, egg release directly into the gut is by far the commonest strategy in all (one-, two- and three-host) cycles. This pattern is particularly prevalent in one-host cycles. When eggs are released away from the gut, this is almost always into some lumen that communicates with the gut or the exterior, and rarely direct from the skin to the exterior. Tissue migration before egg release is also common in definitive hosts of multi-host cycles, but relatively less frequent than in definitive hosts in one-host cycles. While all 239 species in intermediate hosts for which the data were available showed tissue migration (i.e. IT; no cases were found for exploitation of the gut, IG), out of 56 species with paratenic hosts that could be scored, 8 (14\%) appeared to be PG rather than PT (Table A1b).

It is well known that other helminth groups (trematodes, cestodes, acanthocephalans) are also dominated by the habit of egg release in the definitive host gut (Chubb et al. in prep.) and 
by larval stages that exploit the tissues of their intermediate hosts. In Section 3 we construct models to derive theoretical conditions for this pattern to evolve, and in Section 6.1, discuss how this pattern may arise from the advantages of the gut as a place to release eggs, and the benefits of the tissues for increased growth and possibly reduced mortality.

\section{Theoretical Analysis}

We used a simplified notation based on the results in Appendix A, Table A1b to make the theoretical analysis tractable. Thus if strategy $G$ is the first or the only strategy in a host (e.g. IG, DG), it implies that the parasite grows and develops in the gut, or if $\mathrm{G}$ is the final strategy in a definitive host (e.g. DTG), egg release is in the gut of a given host. T is its equivalent for the tissues, and implies a tissue migration and growth phase, or if $\mathrm{T}$ is the final strategy (e.g. DT) in a definitive host, egg release occurs at some site away from the gut. Again, for trophic transmission, transient passage in the gut lumen before penetrating the wall was not counted as an initial $\mathrm{G}$ phase. These simplified definitions are outlined in Table 1. Some of these strategies do not occur in nematodes (or other helminth groups), but are included for theoretical comparison.

For one-host life cycles of vertebrate nematodes, both DG and DTG are common, though much more rarely we find DT (classified as $\mathrm{DT}_{\mathrm{eO}}$ or $\mathrm{DT}_{\mathrm{eS}}$ in Appendix A). For two-host cycles the commonest strategies are IT-DG and IT-DTG, though these are by no means the only patterns, e.g. Stephanurus dentatus shows PT-DT (more fully, PT- DT $\mathrm{eO}_{\text {) }}$ in its two-host life cycle, Anderson 2000). Such patterns suggest that evolution can indeed generate deviations from the 'intermediate host - tissues', 'definitive host - gut' (i.e. IT-DG, or ITDTG) system. We here seek to explain why the IT-DG (or IT-DTG) life history, long seen as axiomatic, is so ubiquitous, particularly in trophically transmitted helminths. For simplicity, 
we confine our analysis to trophic transmission, though it can readily be extended to transmission by skin penetration (see Section 6.3), and yields similar conclusions.

\section{The one-host cycle}

In Section 6.1 (see also Chubb et al. submitted) we argue that relative to the tissues, the gut appears to have overwhelming advantages for propagule release, but that it is less favourable for growth, and possibly also for survival. Thus in a definitive host, there may be advantages in migrating to the tissues to grow, and migrating back to the gut at maturity to release the eggs if the costs of migration (which may be significant; Read \& Skorping 1995, Mulcahy et al. 2005) are sufficiently small.

Nematodes (Appendix A, Table A1b) show the following general strategies in definitive hosts: DG, DT, and DTG, but not DGT. If growth and mortality rates are constant (but see Section 6.4), it is clear that growth should occur only in one site, for the following reason (see Fig. 2). Once establishment has occurred, there are four important life history parameters: growth rate, mortality rate, transmission rate, and reproduction. If for all parameters, one site (gut or tissues) is always better, there is no reason to switch from that site. But if one site is better initially for one parameter, and another location better later for a second parameter, then switching may be favoured. As is common in life history analysis, we show that growth and mortality typically act as a conjoint parameter, the ratio of growth rate/mortality rate. Transmission rate is likely to be equal from both sites. Thus for definitive hosts, if growth rate/mortality rate is higher in the tissues, it may pay to migrate to the tissues for growth and return to the gut for propagule release (Fig. 2a). For intermediate hosts, higher growth rate/mortality rate in the tissues may also favour migration, but for paratenic hosts, in which by definition there is no growth, the important comparison is simply the mortality rates in the two sites (Fig. 2b). 
If our hypothesis is correct about the relative merits of the gut and tissues for growth, survival and propagule release (see Section 6.1), the strategy DGT is much less likely to be favoured. The commonest strategies for single host cycles are indeed DG and DTG (Appendix A, Table A1b).

\subsection{Assumptions and notation}

Our analysis and notation is developed from that of Ball et al. (2008), which gives a full glossary of terms.

For one-host cycles, the parasite grows and reproduces in the definitive host, which we call host $\mathrm{j} . P_{0 j}$ is the probability that the helminth propagule survives to be ingested by $\mathrm{j}$, and $P_{e j}$ is the probability that the parasite entering $\mathrm{j}$ successfully establishes in $\mathrm{j}$.

For simplicity, it is assumed that after establishment, the larval helminth grows linearly (see also Ball et al. 2008, Parker et al. 2009) at rate $g_{j}\left(g_{G j}\right.$ in the gut, $g_{T j}$ in the tissues) starting from an initial size of $W_{0}$ at the time of establishment, $t=0$. Growth stops at sexual maturity at time $t_{j}^{*}\left(t_{G j}^{*}\right.$ in the gut, $t_{T j}^{*}$ in the tissues) at size $W_{j}^{*}\left(W_{G j}^{*}\right.$ in the gut, $W_{T j}^{*}$ in the tissues, or on return to the gut providing all growth has occurred in the tissues). Its size at time $t<t_{j}^{*}$, is $W(t)=W_{0}+g_{j} t\left(t<t_{j}^{*}\right)$. The parasite sustains a constant mortality rate of $p_{j}$ ( $p_{G j}$ in the gut, $p_{T j}$ in the tissues).

Migration to or from the tissues in $\mathrm{j}$ is assumed to have a cost (see Read \& Skorping 1995, Mulcahy et al. 2005) that we model by means of a factor $M_{j}\left(0 \leq M_{j} \leq 1\right)$, which is most easily interpreted as the probability of surviving the migration. We use subscripts to define the migration survival probabilities, depending on the direction of the migration. The subscript order indicates first the parasite's site at (i) the start and (ii) the end of migration (regardless of the time spent in a site), and then (iii) defines the host. For example, if the 
larva migrates immediately after establishment from the gut to the tissues in host $\mathrm{j}$, its probability of survival on arrival to its site in the tissues would be $M_{G T j}$.

We assume that reproductive rate is proportional to adult body size (see Poulin 2007; typically, female egg production is linearly related to body mass), where $b$ is the constant of proportionality ( $b_{G j}$ in the gut, $b_{T j}$ in the tissues). If reproduction occurs in the tissues, eggs are typically released into some lumen that the tissue is associated with, e.g. lung or bladder, and thence to the exterior, or in a few taxa, to the exterior directly. The expected length of adult life is $1 / p_{j}$, and hence the expected number of eggs viewed from the age of sexual maturity is $b_{j} W_{j}^{*} / p_{j}$.

\subsection{Analysis}

We first compare the two common strategies for trophically transmitted nematodes, DG and DTG. These strategies would follow the common pattern that a free-living propagule is ingested by the definitive host, growth to sexual maturation and egg release occur in the host gut (DG), or there is an immediate migration from the gut to the tissues, followed by growth to the adult size (our model assumes all growth to be in the tissues, but see Section 6.4), followed by return to the gut for egg release (DTG).

Consider first the strategy of staying in the host gut, DG. Since we assume constant mortality rates $p_{G j}, p_{T j}$, the rate of change in probability $n$ that the parasite is still alive in the gut is $d n / d t=-p_{G j} n$, and the expected fitness (number of eggs) of the DG strategy will be

$$
E(\mathrm{DG})=P_{0 j} P_{e j} e^{-p_{G j} t_{G j}^{*}}\left(b_{G} W_{G}^{*} / p_{G j}\right) .
$$

The fitness of the DTG strategy, remembering that the transient gut phase immediately after infection is insignificant in terms of growth or mortality (other than that already included 
in the establishment probability, $\left.P_{e j}\right)$, that it undergoes two migrations, and that it achieves the size for sexual maturity in the tissues but lays its eggs in the gut, is

$E(\mathrm{DTG})=P_{0 j} P_{e j} M_{G T j} e^{-p_{T j} t_{T j}^{*}} M_{T G j}\left(b_{G} W_{T}^{*} / p_{G j}\right)$.

From our earlier work (e.g. Parker et al. 2003) we know that

$W_{j}^{*}=g_{j} / p_{j}$

$t_{j}^{*}=1 / p_{j}-W_{0} / g_{j}$

Thus for $E(\mathrm{DTG})>E(\mathrm{DG})$, we need

$M_{G T j} M_{T G j} e^{-p_{T j} t_{T j}^{*}} W_{T}^{*}>e^{-p_{G j} t_{G j}^{*}} W_{G}^{*}$

and substituting from (3a), (3b), this becomes

$\ln \left\lfloor M_{G T j} M_{T G j}\left(\frac{g_{T j} p_{G j}}{g_{G j} p_{T j}}\right)\right\rfloor>W_{0}\left(p_{G j} / g_{G j}-p_{T j} / g_{T j}\right)$.

From (4a), the conditions that favour tissue migration in a definitive host are: (i) low costs of migration, i.e. high survival probability $M_{G T j} M_{T G j}$, (ii) small size at infection, $W_{0}$,

(iii) low growth rate in the gut relative to the tissues $\left(g_{G j}<g_{T j}\right)$, and (iv) high mortality rate in the gut relative to the tissues $\left(p_{G j}>p_{T j}\right)$. Condition (4a) shows the importance of the growth rate/mortality ratios (Fig. 1a); the site with the higher $g_{j} / p_{j}$ ratio has the higher probability of exploitation if migration costs are sufficiently small.

Size data for mature worms are much more readily obtainable from the literature than are growth and mortality data. We can rewrite (4a) as

$\ln \left[M_{G T j} M_{T G j}\left(\frac{W_{T}^{*}}{W_{G}^{*}}\right)\right]>W_{0}\left(1 / W_{G}^{*}-1 / W_{T}^{*}\right)$.

Because $M_{G T j} M_{T G j}<1$, this condition cannot be satisfied unless $W_{T}^{*}$ is sufficiently greater than $W_{G}^{*}$ (in which case the 'stay in the gut' strategy, DG, is favoured). Some numerical results are shown in Fig. 3; DTG is favoured if the value of $W_{T}^{*}$ lies above a given line representing equality in (4b); below a given line, DG is favoured. The lower the chances of 
surviving migration to the tissues and returning to the gut $\left(M_{G T j} M_{T G j}\right)$, the higher the threshold line in Fig. 3. So, for comparable helminths with one-host cycles that release their eggs into the gut, we might expect those showing the DTG strategy to mature at larger body size than those that show the DG strategy. Exactly this pattern has been shown for nematodes by Read \& Skorping (1995).

What of the strategies for migration from the gut, with maturation and egg release away from the gut, i.e. strategies DT, DGT? While DGT is not found, the strategy DT is not uncommon (see Appendix A, Table A1b) because egg release can occur via some other host lumen that communicates directly with the exterior or indirectly via the gut (i.e. $T_{m O}, T_{e O}$ ), or in rare instances, into the host soma itself $\left(T_{e S}\right)$. Such strategies remove the cost of return migration to the gut, but probably offer lower reproductive rates through reduced efficiency or prospects for egg release: we therefore suggest that there is a lower zone of evolutionary attraction for DT and DGT strategies because $b_{G} W_{G}^{*} / p_{G j}>b_{T} W_{T}^{*} / p_{T j}$

\section{The two-host case}

The two-host case is much more complex because (for a two-host cycle) the parasite transfers from an intermediate host $\mathrm{i}$ to the definitive host $\mathrm{j}$; thus even if we confine our analysis to trophic transmission there are many possible strategies. In the intermediate host, there is commonly a growth phase followed by growth arrest (i.e. growth arrest at larval maturity or GALM; Ball et al. 2008, Parker et al. 2009), which may be accompanied by encapsulation or encystment. However, as we shall see, the conclusions remain rather similar to those for just one host.

\subsection{Assumptions and notation}


We again base our analysis on the approach of Ball et al. (2008) and Parker et al. (2009) for transfer between hosts $\mathrm{i}$ and $\mathrm{j}$ by trophic transmission. Following the one-host analysis, we use $i$ and $j$ as subscripts to define the intermediate (I) and the definitive (D) host, and G and $\mathrm{T}$ as subscripts to define the site in the host.

Consider first parameters relating to intermediate host i. By analogy with the one-host case, $P_{0 i}$ is the probability that the helminth propagule survives to be ingested by i, and $P_{e i}$ is the probability that the parasite entering i successfully establishes in i. After establishment the parasite grows until GALM occurs at time $t_{d}$, so there are two mortality rates in host i: $p_{i}^{1}$ for $t<t_{d}$, $p_{i}^{2}$ for $t \geq t_{d}$,

which are the constant mortality rates before and after GALM. Parker et al. (2009) suggested that typically $p_{i}^{1}>p_{i}^{2}$. Again, growth is linear, at rate $g_{i}$, and the initial size is $W_{0}$ at $t=0$, the time of establishment in i. Size at time $t<t_{d}$ is $W(t)=W_{0}+g_{i} t$; after $t_{d}$ the larva remains at size $W_{d}$ until it dies or transfers to host $\mathrm{j}$. Within host $\mathrm{i}$, the parasite has a constant transmission rate, $p_{i j}$, to host $\mathrm{j}$. For trophic transmission, this is the rate at which the intermediate host is eaten by the definitive host.

With trophic transmission, the optimal time of GALM, $t_{d}$, cannot be defined unless mortality rates increase with time (Ball et al. 2008, Parker et al. 2009). Thus the simplifying assumption that mortality rates, $p_{i}^{1}, p_{i}^{2}$, are constant relates to the supposition that if growth continues beyond time $t>t_{d}$, mortality rate quickly becomes infinite, so that selection favours GALM at $t_{d}$, as assumed by Parker et al. (2003). For our present purposes, this assumption allows simple conditions to be derived analytically for the different host exploitation strategies.

In earlier analyses (Ball et al. 2008, Parker et al. 2009), we took fitness (total eggs produced) as the sum 
$E=w_{1}+w_{2}$,

where $w_{1}=$ the number of eggs produced by the parasite if it is ingested by $\mathrm{j}$ before $t_{d}$ and $w_{2}=$ eggs produced if it is ingested after $t_{d}$. If the parasite is ingested before $t_{d}$, it has reached size $W_{0}+g_{i} t$ in host $\mathrm{i}$, so that the time taken to reach maturity in host $\mathrm{j}$ is $\left(W_{j}^{*}-W_{0}-g_{i} t\right) / g_{j}$; if ingested after $t_{d}$ the time taken to reach maturity in host $\mathrm{j}$ is $\left(W_{j}^{*}-W_{d}\right) / g_{j}$, where $W_{d}=W_{0}+g_{i} t_{d}$ is the size at GALM. In many helminths, there is little probability of successful establishment in $\mathrm{j}$ if ingested much before $t_{d}$, with a rapid increase in probability around $t_{d}$.

For parasites that do not grow at all in the first (i.e. paratenic) host, $w_{1}=0$ because $t_{d}=$ 0 , and hence $W_{d}=W_{0}$. Thus $w_{2}$ defines the fitness exactly for paratenic hosts.

For cases where there is growth in the first (i.e. intermediate) host, we make the approximation that $w_{1}=0$, i.e. that the probability of establishment in $\mathrm{j}$ is zero until immediately before GALM, and $P_{e j}$ immediately after GALM. All parasites establishing in j have, on this approximation, an initial size $W_{d}$, as in the paratenic case (where $W_{d}=W_{0}$ ). An alternative approximation is explored in Appendix B.

The probability the parasite is still alive at time $t$ is $n(t)$. Ball et al. (2008, Eq. (13)) showed that

$w_{2}=A e^{p_{j} g_{i} t_{d} / g_{j}} n\left(t_{d}\right) / p_{i}^{d} ;$

where $A \equiv\left(b W_{j}^{*} / p_{j}\right) p_{i j} P_{e j} e^{-\left(W_{j}^{*}-W_{0}\right) p_{j} / g_{j}}$ (Ball et al. 2008, Eq. (10)), and $p_{i}^{d}=p_{i}^{1}+p_{i j}$ (note that there is an error in the definition of $p_{i}^{d}$ in Table 1, Ball et al. 2008).

Then, assuming that $d n / d t=-\left(p_{i}^{1}+p_{i j}\right) n$ for $t<t_{d}, n\left(t_{d}\right)=n(0) e^{-\left(p_{i}^{1}+p_{i j}\right) t_{d}}$, $E=w_{2}=\left(A e^{p_{j} g_{i} t_{d} / g_{j}} P_{0 i} P_{e i} e^{-\left(p_{i}^{1}+p_{i j}\right) t_{d}} / p_{i}^{d}\right)$

where $P_{0 i}$ is the probability that the helminth propagule survives to be ingested by $\mathrm{i}$, and $P_{e i}$ is the probability that the parasite entering i successfully establishes in i. Note that in 5(a) and 
5(b), the variables can be further defined with reference to the site in which growth occurs, e.g. in the tissues, $p_{T i}^{d}=p_{T i}^{1}+p_{T i j}$, and in the gut, $p_{G i}^{d}=p_{G i}^{1}+p_{G i j}$.

We can now extend equation (5b) for $E$ under trophic transmission to include different $G$ and $\mathrm{T}$ strategies by adding the appropriate subscripts, following the convention for one-host cycles.

\subsection{Strategies in the intermediate host}

Our analysis of Anderson (2000) for nematodes of vertebrates showed that tissue migration was ubiquitous in intermediate hosts (Appendix A, Table A1b). For trophic transmission, the transient gut phase between infection and tissue migration in intermediate hosts was not recorded as a $\mathrm{G}$ phase (as with definitive hosts). Although in some paratenic hosts it is clear that the larva remained in the gut (which is recorded as a G phase), this was never reported for intermediate hosts. Complex strategies involving return to the gut (ITG) or growth in the gut followed by migration to the tissues (IGT) were never reported, and we know of none in other groups, so we must assume that conditions favouring ITG or IGT are rare or absent. The probable reason for the absence of ITG (c.f. the ubiquity of DTG; Appendix A, Table A1b) is that since no propagule release occurs in intermediate hosts, there is no reason for a return migration to the gut. The selective pressures shaping the site of exploitation are dominated by growth and mortality rates which act as a single variable (Fig. 2a); whichever site is initially the more favourable (after discounting migration costs) is likely to remain so.

We therefore focus on the condition for the strategy IT to be of advantage over IG. With IT, the larva grows in the tissues to GALM, and remains there after GALM. We consider separately the condition favouring tissue exploitation up to GALM, then the condition favouring staying there after GALM. 
For migration to and subsequent growth in the tissues to be favourable, the probability of surviving to reach GALM size, $W_{d}$, must be greater in the tissues than the gut after discounting the migration costs, i.e.

$$
M_{G T i} P_{0 i} P_{e i} e^{-\left(p_{T i}^{1}+p_{T i j}\right) t_{T d}}>P_{0 i} P_{e i} e^{-\left(p_{G i}^{1}+p_{G i j}\right) t_{G d}},
$$

where times $t_{T d}$ and $t_{G d}$ are the times to reach GALM in the tissues and gut of host $\mathrm{i}$ respectively.

Since $t_{d}=\left(W_{d}-W_{0}\right) / g_{i}$, where $g_{i}$ is either $g_{T i}$ or $g_{G i}$, (6a) becomes

$\ln M_{G T i}>\left(W_{d}-W_{0}\right)\left\{\left(p_{T i}^{1}+p_{T i j}\right) / g_{T i}-\left(p_{G i}^{1}+p_{G i j}\right) / g_{G i}\right\}$.

It is interesting that $\left(p_{i}^{1}+p_{i j}\right) / g_{i}$ is equivalent to $1 / W_{i}^{*}$, where $W_{i}^{*}$ is the size that the parasite in host $\mathrm{i}$ would have matured sexually were it to have used $\mathrm{i}$ as a definitive host; c.f. (4b).

If migration costs are trivial, $M_{G T i} \rightarrow 1$, tissue migration simply requires that $g_{T i} /\left(p_{T i}^{1}+p_{T i j}\right)>g_{G i} /\left(p_{G i}^{1}+p_{G i j}\right)$.

This is equivalent to the growth rate/mortality rate ratio rule outlined in Fig. 1a. Since the growth rate/mortality rate ratio is likely to be higher in the tissues (see Section 6.1), it is perhaps unsurprising that tissue migration in intermediate hosts is so ubiquitous.

To derive the condition for selection to favour staying in the tissues after GALM, we use (5a) with $n\left(t_{d}\right)=1.0$, since the larva has survived to time $t_{d}$ :

$$
w_{2}=A_{U} e^{p_{j} g_{i} t_{d} / g_{j}} / p_{i}^{d}
$$

in which $g_{i}=g_{T i}$ (since growth has occurred in the tissues), and $p_{i}^{d}=p_{T i j}+p_{T i}^{2}$ if the larva remains in the tissues, or $p_{G i j}+p_{G i}^{2}$ if the larva migrates to the gut. Thus for staying in the tissues to be favoured,

$$
A_{U} e^{p_{j} g_{T i} t_{T d} / g_{j}} /\left(p_{T i j}+p_{T i}^{2}\right)>M_{T G i} A_{U} e^{p_{j} g_{T i} t_{T d} / g_{j}} /\left(p_{G i j}+p_{G i}^{2}\right)
$$

where $M_{T G i} \leq 1$ is the probability of surviving the migration from the tissues to the gut. In term $A_{U}$, the sites occupied in $\mathrm{j}$ can be left undefined (they are independent of the 'decision' 
to migrate in i), except for $p_{i j}\left(p_{i j}=p_{T i j}\right.$ if transmission to $\mathrm{j}$ occurs from the tissues of $\mathrm{i}$;

$p_{i j}=p_{T i j}$ if transmission occurs from the gut of i). Hence

$\left(p_{G i j}+p_{G i}^{2}\right) /\left(p_{T i j}+p_{T i}^{2}\right)>M_{T G i} \frac{p_{G i j}}{p_{T i j}}$.

If migration costs are trivial $\left(M_{T G i} \rightarrow 1\right)$ and transmission prospects equal in the gut and tissues $\left(p_{G i j}=p_{T i j}\right)$, then remaining in the tissues after GALM simply requires that

$p_{G i}^{2}>p_{T i}^{2}$

which may be expected if the gut is more likely to be a hostile environment for the larva than the tissues (see Section 6.1.2), in which the larva may be isolated from host responses after GALM by encapsulation or encystment (Parker et al. 2009). Result (7b) is unsurprising since there is no further growth after GALM and propagules release is not an issue, then the only relevant parameter becomes the mortality rate (as in Fig. $2 b$ ).

In summary, IT is likely to be favoured over IG through (6a), which favours migration to the tissues for growth to GALM, and (7a), which favours remaining there after GALM. IG appears plausible only when the prospects of surviving migration to the tissues $\left(M_{T G i}\right)$ are low.

\subsection{Strategies in the definitive host}

Table A1b (Appendix A) shows that in nematodes with complex life cycles, in their definitive hosts the simple strategy of exploiting the gut lumen (DG) is common, as would be typically the case for acanthocephalans and cestodes (or in very few cestodes, the bile ducts). The complex strategy of an immediate tissue migration followed by growth and a return to the gut (DTG) is quite common in nematodes; in contrast, the strategy DGT was never seen. This parallels the situation for one-host life cycles, which we have already analysed. 
Once the parasite has entered the definitive host, the conditions favouring DTG over DG are analogous to those for one-host cycles, so remembering that the parasite enters $\mathrm{j}$ at size $W_{d}$ (rather than $W_{0}$,) we can rewrite (4a) and (4b) as

$\ln \left\lfloor M_{G T j} M_{T G j}\left(\frac{g_{T j} p_{G j}}{g_{G j} p_{T j}}\right)\right\rfloor>W_{d}\left(p_{G j} / g_{G j}-p_{T j} / g_{T j}\right)$,

i.e. $\ln \left[M_{G T j} M_{T G j}\left(\frac{W_{T j}^{*}}{W_{G j}^{*}}\right)\right\rfloor>W_{d}\left(1 / W_{G j}^{*}-1 / W_{T j}^{*}\right)$.

Thus conclusions remain as for the one-host case, that tissue migration is favoured when migration costs are low, size at infection is small, growth rate in the gut is low relative to the tissues, and mortality rate in the gut is high relative to the tissues.

Further, we can make parallel conclusions about the rarity of DT and DGT strategies, which are less likely because of problems of egg release.

\subsection{Why is IT-DG (or IT-DTG) so ubiquitous?}

We next seek to explain why the IT-DG strategy has become ubiquitous in complex life cycles of helminths. We focus on IT-DG for simplicity, but can readily extend the analysis to IT-DTG (which is also common in nematodes; Appendix A, Table A1b) by application of (8a), (8b).

We therefore seek to explain why $E(\mathrm{IT}-\mathrm{DG})>E(\mathrm{IT}-\mathrm{DT}), E(\mathrm{IG}-\mathrm{DT}), E(\mathrm{IG}-\mathrm{DG})$

i.e. the fitness of the IT-DG strategy is greater than that of the other three possible simple strategies for the two-host cycle.

Since there appear to be major benefits to releasing propagules in the gut (Section 6.1.3; Chubb et al. in prep.), we dismiss IT-DT and IG-DT, and seek to explain simply why 
$E(\mathrm{IT}-\mathrm{DG})>E(\mathrm{IG}-\mathrm{DG})$, i.e. why larval parasites avoid the gut of their intermediate hosts.

From (5b) we can see that

$$
\begin{aligned}
& E(\mathrm{IT}-\mathrm{DG})=w_{2}(\mathrm{IT}-\mathrm{DG})=A_{G} M_{G T i} e^{p_{G j} g_{T i} t_{T d} / g_{G j}} P_{0 i} P_{e i} e^{-\left(p_{T i}^{1}+p_{T i j}\right) t_{T d}} /\left(p_{T i j}+p_{T i}^{2}\right), \\
& E(\mathrm{IG}-\mathrm{DG})=w_{2}(\mathrm{IG}-\mathrm{DG})=A_{G} e^{p_{G j} g_{G i} t_{G d} / g_{G j}} P_{0 i} P_{e i} e^{-\left(p_{G i}^{1}+p_{G i j}\right)_{G d}} /\left(p_{G i j}+p_{G i}^{2}\right),
\end{aligned}
$$

in which, for both cases,

$$
A_{G}=p_{i j} P_{e j}\left(b_{G j} W_{G j}^{*} / p_{G j}\right) e^{-p_{G j}\left(W_{G j}^{*}-W_{0}\right) / g_{G j}}
$$

but in (9), $p_{i j}=p_{T i j}\left(\right.$ transmission occurs from the tissues of i) and in (10) $p_{i j}=p_{T i j}$

(transmission occurs from the gut of i). Note that we have added the term $M_{G T i}$ to account for the costs of tissue migration in the intermediate host; there is no cost of return migration since the larva remains in the tissues at GALM to be consumed by host $\mathrm{j}$.

Thus $E(\mathrm{IT}-\mathrm{DG})>E(\mathrm{IG}-\mathrm{DG})$ if

$\ln \left\lfloor M_{G T i} \frac{p_{T i j}}{p_{G i j}}\left(\frac{p_{G i j}+p_{G i}^{2}}{p_{T i j}+p_{T i}^{2}}\right)\right\rfloor>\left(p_{G j} / g_{G j}\right)\left(g_{G i} t_{G d}-g_{T i} t_{T d}\right)+\left(p_{T i}^{1}+p_{T i j}\right) t_{T d}-\left(p_{G i}^{1}+p_{G i j}\right) t_{G d}$.

Assuming that transmission is independent of site $\left(p_{G i j}=p_{T i j}\right)$, condition (12a) is likely to be satisfied if (i) mortality is higher in the gut $\left(p_{G i}^{1}>p_{T i}^{1}, p_{G i}^{2}>p_{T i}^{2}\right)$, and (ii) growth rate is lower in the gut $\left(g_{G i}<g_{T i}\right)$ so that $t_{G d}>t_{T d}$, provided that the (iii) mortality costs of migration $\left(1-M_{T G i}\right)$ are sufficiently small.

We can write (12a) in terms of body size as

$$
\ln \left\lfloor M_{G T i} \frac{p_{T i j}}{p_{G i j}}\left(\frac{p_{i j}+p_{G i}^{2}}{p_{i j}+p_{T i}^{2}}\right)\right\rfloor>\frac{\left(W_{G d}-W_{T d}\right)}{W_{j}^{*}}+\frac{\left(p_{T i}^{1}+p_{i j}\right) W_{T}}{g_{T i}}-\frac{\left(p_{G i}^{1}+p_{i j}\right) W_{G}}{g_{G i}},
$$

where $W_{T}=W_{T d}-W_{0}$ and $W_{G}=W_{G d}-W_{0}$, i.e., measures of the total growth in host i.

Further interpretation of (12a) depends on the ancestral state. If ancestrally the parasite lived in host i's gut (as might be expected from recent theories for the evolution of complex life cycles; Parker et al. 2003, 2009, then (12a) can be seen as the condition for the spread of a 
mutant performing tissue migration. If this mutant is programmed such that size at GALM, $W_{d}$, remains fixed at the same as the rest of the population, $g_{G i} t_{G d}=g_{T i} t_{T d}=W_{d}-W_{0}$. Then $\ln \left\lfloor M_{G T i} \frac{p_{T i j}}{p_{G i j}}\left(\frac{p_{i j}+p_{G i}^{2}}{p_{i j}+p_{T i}^{2}}\right)\right\rfloor>\left(W_{d}-W_{0}\right)\left\{\left(p_{T i}^{1}+p_{T i j}\right) / g_{T i}-\left(p_{G i}^{1}+p_{G i j}\right) / g_{G i}\right\}$.

Since $\left(p_{i}^{1}+p_{i j}\right) / g_{i}$ is equivalent to $1 / W_{i}^{*}$, where $W_{i}^{*}$ is the size that the parasite would have matured sexually in host $\mathrm{i}$ were it to have used $\mathrm{i}$ as a definitive host; c.f. (4b). Condition (12c) for tissue migration in intermediate hosts generates similar conclusions to (4a) and (8a) for tissue migration in definitive hosts. Since the right hand size is likely to be negative, (12c) shows that, all else equal, tissue migration in the intermediate host is more likely to occur in species that have, in intermediate host i (i) high increase in body mass, $\left(W_{d}-W_{0}\right)$, (ii) high probability, $M_{G T i}$, of surviving migration to the tissues, (iii) lower mortality rates in the tissues, and (iv) higher growth rates in the tissues than the gut.

Fig. 4 examines the threshold value for $M_{G T i}$, the probability of surviving tissue migration, against the total growth up to GALM in host $\mathrm{i}\left(W_{d}-W_{0}\right)$, from $(12 \mathrm{c})$. In the parameter space above each line, strategy IT-DG is favoured; below each line strategy IG-DG is favoured. Thus the greater the mass increase in $\mathrm{i}\left(W_{d}-W_{0}\right)$, the more likely that tissue migration will be favoured, and increasingly so as the growth rate in the tissues increases above that in the gut (compare Fig. $4 \mathrm{a}, \mathrm{b}$ ). When mortality is equal in the gut and tissues (upper continuous curves in Figs. 4a, b), conditions favouring IT-DG are most restrictive; decreasing the mortality rate in the tissues (lower continuous curves in Figs. 4a, b) increases the chances of tissue migration in the intermediate host. The effect of GALM is complex: if mortality reduces proportionately in both sites at GALM, there is no effect on the line if mortality rates are equal in the two sites. However, if mortality in the tissues is lower, the same proportionate decrease in mortality rate at GALM increases the threshold line (see broken curves), making the condition for spread of tissue migration in i more restrictive. However, if GALM results in a mortality decrease only in the tissues (dotted curves) as seems 
more probable (see Section 6.1.2), this decreases the threshold line, facilitating the evolution of tissue migration in $\mathrm{i}$.

The alternative, possibly less plausible, scenario is that the mutant is programmed such that time at GALM, $t_{d}$, remains fixed, i.e. $t_{T d}=t_{G d}=t_{d}$, so that (12a) becomes

$$
\ln \left\lfloor M_{G T i} \frac{p_{T i j}}{p_{G i j}}\left(\frac{p_{i j}+p_{G i}^{2}}{p_{i j}+p_{T i}^{2}}\right)\right\rfloor>\left[\left(p_{G j} / g_{G j}\right)\left(g_{G i}-g_{T i}\right)+\left(p_{T i}^{1}-p_{G i}^{1}\right)\right] t_{d},
$$

which again yields very similar conclusions. It can be written in terms of body size as

$$
\ln \left\lfloor M_{G T i} \frac{p_{T i j}}{p_{G i j}}\left(\frac{p_{i j}+p_{G i}^{2}}{p_{i j}+p_{T i}^{2}}\right)\right\rfloor>\left(W_{G d}-W_{T d}\right) / W_{j}^{*}+\left(p_{T i}^{1}-p_{G i}^{1}\right) t_{d}
$$

where $W_{G d}, W_{T d}$ are the sizes achieved at GALM in the gut and tissues respectively. Since the right hand side of (12d) is likely to be negative, all else equal, tissue migration in the intermediate host is more likely to occur in species that have low body mass at sexual maturity, in definitive host $\mathrm{j}$.

The main difference between the two assumptions about the ancestral state is that if size at GALM is fixed (12c), invasion of tissue migration in host $i$ is independent of growth and mortality parameters in host $\mathrm{j}$ (which define maturity size $W_{j}^{*}$ ); in contrast, if the time at which GALM occurs is fixed (12d), invasion of tissue migration in host $\mathrm{i}$ is dependent on mortality and growth rates in both hosts.

In conclusion, conditions for tissue migration in intermediate hosts, i.e. $E(\mathrm{IT}-\mathrm{DG})>E(\mathrm{IG}-\mathrm{DG})$, are rather robust against assumptions about ancestral states, requiring (i) low costs of tissue migration, (ii) higher growth rate in the tissues, and (iii) higher mortality rate in the gut. These parallel the conditions for tissue migration in definitive hosts in one-host cycles, i.e. $E(\mathrm{DTG})>E(\mathrm{DG})$.

\subsection{Paratenicity}


If the first host $\mathrm{i}$ is a paratenic host, there is no growth and $W_{d} \equiv W_{0}$ (see Parker et al. 2009). Following the section above, we can ask whether migration to the tissues will be favoured in the paratenic host, i.e. whether $E(\mathrm{PT}-\mathrm{DG})>E(\mathrm{PG}-\mathrm{DG})$. In the case of paratenicity, use of $w_{2}$ alone is exact (there is no period before $t_{d}$ and hence $w_{1}=0$ ). Thus we can use (5a) with $n(0)=1.0$ and $t_{d}=t_{0}$; hence

$$
\begin{aligned}
& E(\mathrm{PT}-\mathrm{DG})=A_{G} M_{G T i} P_{0 i} P_{e i} /\left(p_{i j}+p_{T i}^{2}\right), \\
& E(\mathrm{PG}-\mathrm{DG})=A_{G} P_{0 i} P_{e i} /\left(p_{i j}+p_{G i}^{2}\right),
\end{aligned}
$$

where $A_{G}$ is given in (11) with $p_{i j}=p_{T i j}$ when transmission occurs from the tissues of $\mathrm{i}$, and $p_{i j}=p_{G i j}$ when transmission occurs from the gut of $i$. Thus for migration to the tissues to be favoured in the paratenic host,

$$
M_{G T i} \frac{p_{T i j}}{p_{G i j}}>\left(p_{T i j}+p_{T i}^{2}\right) /\left(p_{G i j}+p_{G i}^{2}\right),
$$

c.f. condition (7a) for the larva to stay in the tissues after GALM. If migration costs are trivial $\left(M_{G T i} \rightarrow 1\right)$, and transmission equal across sites $\left(p_{G i j}=p_{T i j}\right)$, we need simply that $p_{T i}^{2}>p_{G i}^{2}$ (Fig. 1b).

\section{Discussion}

The survey of extant taxa of nematodes (Appendix A, Table A1b) based on Anderson (2000) provides an empirical comparison with the model predictions. Our analysis shows that the typical IT-DG and IT-DTG patterns of parasitic helminths can readily be explained by higher growth benefits (e.g. due to richer potential food source) and possibly higher survival benefits (e.g. due to avoidance of mechanical loss in gut due to peristalsis) in the tissues, with much higher reproductive benefits for propagule release of the gut. In the following sections we consider the differences between gut and tissues for helminth life in more detail, and predict the relative merits for the main life historic parameters (mortality, growth, 
transmission and reproduction) of the gut and tissue alternatives (Table 2), where + indicates the more favourable and - the less favourable option for each factor considered.

\subsection{The relative advantages and disadvantages of gut versus tissue exploitation}

Present day helminths have characteristic bauplans depending on whether they are adapted to live in the gut (e.g. attachment and feeding organs) and/or tissues (e.g. penetration organs for migration and abilities to withstand host immune responses). Trophically transmitted helminths first enter the host gut lumen, and must then either migrate to the tissues or remain in the gut. Those that infect by skin penetration initially enter into the tissues and must subsequently select a site in the tissues or move to the gut.

\subsubsection{Growth rates}

All else equal, the host tissues offer consistent and high quality food compared to a lower quality, semi-digested, and variable quality food in the host gut; this may be a central factor generating higher growth rate benefits in the tissues. Anderson $(1992,2000)$ proposed that species with intermediate hosts (i.e. with typically at least one tissue phase) achieve accelerated growth. A possible disadvantage for growth in the tissues is that local drainage of nutrient resources could occur if the larva remains stationary; however, larvae are often mobile until GALM, after which growth and demand from host tissues cease. Thus local drainage is likely to represent a minor potential disadvantage in view of the high nutrient value of the host tissues relative to the gut contents. In support of the prediction of higher growth rate in the gut, a comparative study of nematodes by Read \& Skorping (1995) found that although the time for development to adulthood remained the same, species with extensive tissue migration phases had on average larger adults than those remaining in the gut, suggesting that those worms with a tissue phase have a higher growth rate in the tissues. 


\subsubsection{Mortality rates}

Mortality rates are of critical importance for all helminth stages. There are several arguments both in favour and against each site (tissues and gut) for survival (see Table 2); it is therefore difficult to deduce their overall difference for this parameter. The two major contrasting considerations would seem to be the adverse effects of peristalsis and other mechanical processes in the gut lumen (which will be minimal in the tissues), and the adverse effects of the host response in the tissues (which will be absent in the gut unless there is contact with, or migration into, the gut mucosa).

The risk of loss due to peristaltic waves or other mechanical effects may constitute a notable factor against gut exploitation for growth. The gut appears to be a dangerous place for the establishment and subsequent survival of helminths (e.g. the cestode Proteocephalus filicollis in Gasterosteus aculeatus in field conditions, with approximately $1 \%$ of the worms present being lost daily, Hopkins 1959, and Echinorhynchus truttae in Salmo (Oncorhynchus) gairdneri and where in experimentally infected fish acanthocephalans at all stages of development were passed with the faeces at irregular intervals, Chubb et al. 1964).

Considering mortality due to host responses, the gut lumen is immunologically inert and hence a priori likely to be safer than the tissues. We include migration into the gut mucosa as mucosal tissue migration ( $\mathrm{T}_{\mathrm{m}}$ in Appendix A, Table A1a, b), but Mulcahy et al. (2005) stress the importance of the adverse immune responses of the host gut mucosa, and suggest that tissue migration beyond the gut mucosa (which we term extensive tissue migration, $\mathrm{T}_{\mathrm{e}}$ in Appendix A, Table A1a, b) may be of selective advantage to helminths to avoid host immunedefence mechanisms evolved to protect the host against threats at the gut mucosal surfaces, i.e. they propose that helminth mortality within the tissues away from the gut would be reduced so as to favour migration from the gut. The gastric mucosa shows protection against mucosal helminth infections by Th2 effector mechanisms (Mulcahy et al. 2005). The 
protective capacity of immune responses against mucosal-dwelling helminths is, in most cases, greater than that against stages dwelling in tissue spaces. Given that the gut lumen is immunologically inert, the best strategies would appear to be to avoid the mucosa and either stay in the gut lumen, or to migrate deep into the tissues. The fact that the mucosa is a site so favoured by nematodes in vertebrate definitive hosts (strategy $\mathrm{DT}_{\mathrm{m}} \mathrm{G}$ in Appendix A, Table A1b) may be seen as an argument against Mulcahy et al.'s proposal. Mulcahy et al. suggest that whereas some helminths have succeeded in minimizing recognition by the host immune system, an alternative strategy might have been migration into tissue spaces (DT $\mathrm{e})$. Our view is that the most salient comparison favouring tissue migration (be it simply mucosal or extensive) is that of the high risks of mechanical loss of small larvae in the gut due to peristalsis, relative to the increased host responses in the tissues. For initial, tissue-invading mutants, the host response in the tissues must have generated significant risks; later, once host-specific resistance had evolved, the costs would relate more to maintaining this resistance.

Parker et al. (2009) suggest that the mortality rate of larvae will be lower when isolated from host responses after GALM by encapsulation or encystment; in our models, this translates into $p_{T i}^{2}<p_{T i}^{1}$. We would also expect the mortality rate of a (hypothetical) growtharrested larva in the gut to be higher than one in the tissues, $p_{G i}^{2}>p_{T i}^{2}$, and possibly equal to or greater than that of an actively growing larva in the gut (i.e. $p_{G i}^{2} \geq p_{G i}^{1}$ ). These asymmetries suggest that condition (12a), favouring the IT-DG over the IG-DG pattern, is likely to be satisfied.

Migration must have significant costs. Read \& Skorping (1995) and Mulcahy et al. (2005) have stressed that migration through host tissues is likely to incur mortality risks, energetic costs, and the costs of adaptation to varying physicochemical environments. For nematodes in definitive hosts, Read \& Skorping (1995) found evidence for lower survival to adulthood in species with tissue migration than species remaining in the gut lumen. The latter 
effect could have been due either to the higher mortality risks involved in migrations to the tissues and return to the gut, i.e. to migration costs per se, or to the mortality rate experienced in the tissue site exploited. Our models most readily allow tissue exploitation to be favoured if both survivorship due to tissue migration $\left(M_{G T j} M_{T G j}\right)$ is high and if mortality rate during development in the tissues $\left(p_{T j}\right)$ is lower than that in the gut $\left(p_{G j}\right)$; for example, see condition (8a). However, provided that growth rates are sufficiently greater in the tissues than the gut, equal or marginally higher mortality through tissue exploitation would not change the prediction that tissue migration should be favoured (see Fig. 2).

We expect that parasite mortality risks through host damage are greater in the tissues than the gut, though exploitation of the body cavity may be relatively harmless to hosts, and hence to helminths, but against this must be set the costs of maintaining position in the gut lumen, which will be considerable compared to the stability afforded by the tissues and body cavity.

In summary, the relative mortality risks of gut versus tissues are hard to assess; our intuition suggests that on balance there is often a marginal advantage for the tissues, but further emipirical evidence would be extremely valuable here.

\subsubsection{Why is the gut so good a site for egg release?}

The gut clearly has a much greater zone of evolutionary attraction than any other site (Appendix A, Table A1b, Chubb et al. submitted) for adults to release eggs. The virtues of a constant flow of faeces to carry propagules out to the exterior for dissemination to next host cannot be overstated and is highly likely to be the reason why so many helminth species have evolved propagule release in the gut, and why in many nematodes of vertebrates there is typically a return migration to the gut after a period in the tissues of definitive hosts.

Adult parasites in the tissues or body cavity have two options for egg release: release in the tissues themselves, or into some lumen in the host soma that communicates either directly or indirectly (usually via the gut) with the exterior. The opportunities for egg release are 
much more restrictive in tissues themselves, and generally require the death of the host for dissemination (e.g. the nematode Calodium hepaticum, releases eggs in the liver parenchyma that are probably disseminated by cannibalism, see Anderson 2000). In rare instances, dissemination is achieved in other ways (e.g. eggs of the trematode Schistosoma mansoni have a spine for cutting through the intestine wall by the action of gut peristalsis).

The vast majority of helminths that reproduce away from the gut release eggs into some lumen, so that eggs can be passed to the exterior. The commonest lumen so exploited in nematodes of vertebrates appears to be the lungs or other parts of the respiratory tract (Anderson 2000), which typically results in propagule release to the exterior via the gut after they are displaced from lungs by host coughing or other responses. A few species discharge eggs into the gall bladder or bilary system, and thence to the gut. Indirect release permits infection of host sites away from the gut but is unlikely to be as effective as direct expulsion into the gut; mortality risks and energetic losses are likely to be greater. The same argument of reduced efficiency is likely to apply for the rather rare instances in which the urino-genital tract is used for egg release. However, some species move (without tissue migration) as preadults from the gut to mature and release eggs in cavities (e.g. lungs) associated with the gut. Why this should be so if the gut is a more effective place for egg release is uncertain.

\subsubsection{Transmission}

Transmission from the free living stage to the first host, or from one host to the next, forms an important life history parameter for helminths, but the theoretical merits of gut and tissues as sites of larvae for transmission are likely to be largely equal. Vertebrate predation usually involves ingestion of the entire body of the prey, and so transmission to the next host is likely to be largely independent of the site (gut or tissues) exploited by larvae. Where ingestion of prey is partial, predators perhaps have preferences for given tissues (e.g. body muscles rather than gut). 


\subsubsection{Summary of relative merits and their significance for the models}

The above arguments, and the summary in Table 2, indicate very strong advantages for reproduction and propagule release in the host gut, which is almost certain to explain the ubiquity of this habit in all helminth groups (Appendix A, Table A1b for nematodes, see Chubb et al. submitted for all helminth groups). For this reason we feel justified in omitting detailed analysis of strategies ending with release of propagules from sites in the tissues (e.g. DT, DGT). Under trophic transmission, for most species, transmission rates to the definitive host are likely to be largely unaffected by residence in the gut or tissues of the intermediate host. Growth rates are potentially higher in the tissues, and there is evidence for this (Read \& Skorping 1995). For mortality rates there are many considerations, making it difficult to predict the relative merits of the two sites; on balance we would envisage less asymmetry between the gut and tissues, perhaps with a marginal bias in favour of the tissues for a majority of species. Some support for this view of relative merits is given in the next section.

\subsection{Model predictions and supporting evidence}

\subsubsection{Gut residence in intermediate versus paratenic hosts}

In support of this view of relative merits, we note that all 239 cases in intermediate hosts that could be categorised from Anderson (2000) showed tissue expoitation (IT) rather than gut residence (IG), but in paratenic hosts (where the effect of growth is removed) 8 (14.3 percent) out of 56 species could be categorised showed gut (PG) rather than tissue residence (PT) (Appendix A, Table A1b). This finding is compatible with a strong benefit for tissues in terms of growth rates for most larval parasites, and a more marginal average benefit across species for the tissues in terms of mortality rates. If migration into the tissues is uncostly ( $M_{G T i} \rightarrow 1$ ), condition (6c) for the intermediate host relies on two parameters, one of which (growth rate) is likely to strongly favour exploitation of the tissues rather than the gut of the 
intermediate host $\left(g_{T i}>g_{G i}\right)$, and another (mortality rate) which is possibly marginally favourable for the tissues (i.e. $p_{G i}^{1}>p_{T i}^{1}$ ). With uncostly migration, residence in the tissues by larvae in paratenic hosts merely requires $p_{G i}^{2}>p_{T i}^{2}$ (condition $(7 \mathrm{~b})$ ), which is more equivocal in view of the difficulty in predicting the relative balance of mortality rates between the tissues and gut.

\subsubsection{Tissue migration in one-host cycles}

Condition (4a) suggests that remaining in the gut for both growth and reproduction (DG) will be favoured if the differences between gut and tissues for the ratio growth rate/ mortality rate are marginal $\left(p_{G j} / g_{G j} \approx p_{T j} / g_{T j}\right)$, if there is a relatively large size at infection $\left(W_{0}\right)$, and/or if there is a relatively low probability of surviving the two migrations $\left(M_{G T j} M_{T G j}\right)$. It would be interesting to examine whether these predictions are met in oxyurid nematodes, where the DG strategy is the rule (bauplan). The opposite conditions, i.e. $g_{T j} / p_{T j}>g_{G j} / p_{G j}$, low $W_{0}$, and high $M_{G T j} M_{T G j}$, favour the common tissue migration strategy (DTG). A prediction of condition (4b), which expresses (4a) in terms of body size, is that those nematodes showing DTG mature at larger body size than those showing DG (see also Fig. 3). This has been shown for nematodes by Read \& Skorping (1995).

\subsubsection{Tissue migration in two-host cycles}

Assuming that transmission rates from the intermediate (or paratenic) hosts to definitive hosts are independent of site (gut or tissues) exploited the infective larva in the intermediate host (i.e. that $p_{T i j} \cong p_{T i j}$ ), predictions for tissue migration are very similar in two-host cycles to those for one-host cycles.

Appendix A, Table A1b suggests that in nematodes, DTG is relatively more common than DG in one-host cycles than in complex cycles. We see an obvious reason for this: the 
larva is bigger in a two-host cycle due to prior exploitation of an intermediate host $\left(W_{d}>W_{0}\right)$, so that if the size at adulthood is not much different in the two cases, condition (4b) for DTG to be favoured in one-host cycles will be easier to satisfy than condition (8b) for two-host cycles. Against this argument might be the expectation that definitive host size could be greater in two-host nematodes. We note, however, that cestodes and acanthocephalans almost always show only IT-DG, and not IT-DTG. Why this should be so in terms of the growth, mortality and size parameters in our models remains to be clarified; some of these helminths grow to a large size in the gut of the definitive host (e.g. the cestode, Diphyllobothrium latum, the acanthocephalan Macracanthorhynchus hirudinaceus), yet others grow little or not at all (e.g. the cestode, Schistocephalus solidus, the acanthocephalan, Acanthocephalus clavula).

We can also ask the related question: why, in two-host cycles, is there apparently always migration to the tissues in intermediate hosts (IT), yet in one host-cycles, though tissue migration (DTG) is very common, many nematodes remain in the host gut (DG)? A reason why tissue exploitation is more likely in the first host of two-host cycles than in a one-host cycle is that there is obviously no need to return to the gut to release eggs in an intermediate host compared to a definitive host. It is easier to satisfy conditions (6b) and (12c) for tissue exploitation intermediate hosts (IT), since they lack the term $M_{T G}$ in condition (4a) for the return tissue migration in the one-host cycle.

\subsection{Bauplan constraints}

Larvae of all trematodes, cestodes, acanthocephalans, and many nematodes penetrate the tissues of their intermediate hosts either from the exterior or through the wall of the gut after ingestion by the host (Chubb et al. submitted). Read \& Skorping (1995) review the classical argument that tissue migrations are 'vestiges' of previous life history patterns, possibly originating either from an ancestral skin penetration mode of infection, and or from the loss of 
the intermediate host in which there was obligate tissue residence. Thus if the intermediate host has been lost (as suggested for some one-host cycles of nematodes, Anderson 2000), tissue migration in the definitive host has been claimed to derive from a genetic bauplan for tissue migration in the lost intermediate host. An implicit assumption in several of the classical claims is that the larva must reside in the tissues of the intermediate host; while this is typically evident (e.g. Appendix A, Table A1b), one must question why. Read \& Skorping, opposing the classical argument, point out that there are many costs to such migrations, and that many nematode species remain in the gut throughout their development even in taxa likely to be derived from ancestors with skin penetration or complex life cycles. We note, however, that the strictly monoxenous oxyurid nematodes, the only nematode group with adult representatives in both vertebrates and invertebrates (Anderson 2000), infect their vertebrate hosts directly by eggs and do not have tissue migrations. The lack of tissue penetration in vertebrate hosts may simply reflect the fact that the ancestral state (in arthropods) did not involve a migration phase. While it is tempting to dismiss the classical view of bauplan constraints, we feel that some caution is prudent; if a series of genetic adaptations to tissue penetration and migration have already evolved, single mutations may not readily suppress migration without resulting in deleterious pleiotropic effects.

\subsection{An anomaly: growth in the gut after an initial tissue phase}

Though our models explain the life history patterns of gut and tissue exploitation by helminths quite well, there is an anomaly that the models do not predict: growth often continues in the gut after the tissue growth phase. The reason for this is that we have assumed growth and mortality rates are to be constant, so that there is an initial 'choice' of best location for growth rate/mortality rate, and then a second 'choice' of best location for egg release. Thus if DTG is favoured, we would expect to see all growth in the tissues, followed 
by migration to the gut for egg release. In fact, most of the nematodes that show the DTG strategy show the majority of their increase in mass following migration to the gut, which we would not predict with our present model of constant growth rate/mortality rate.

In reality, we suspect that size dependent mortality is generally time- or size-dependent (Ball et al. 2008, Lynch et al. 2008, Parker et al. 2009), and unequally so in the gut and the tissues. The complexities of asymmetric time- or size-dependent mortality and growth between gut and tissues are not included in our models. At small size, mortality may be higher in the gut than in the tissues for mechanical reasons such as peristalsis (see Section 6.1.2 and Table 2). In the host tissues, as a parasite's size increases, its mortality rate may accelerate because of its increased host's mortality risk due to the harm inflicted by the large helminth on the host's tissues (Fig. 5a). Despite some notable exceptions, e.g. Dracunculus medinensis, it not common for helminths to achieve large sizes in the tissues. In contrast, in the gut, the parasite's mortality rate may decrease as its size increases because of greater ability to resist peristalsis and other mechanical effects (Fig. 5a). Thus worms that mature at large size may first show an initial tissue growth phase, followed by a further growth phase in the gut, because of the host risks inherent in high growth rate and large size in the tissues. Essentially, there may be a switch during development so that initially, the growth rate/mortality rate ratios are such that $g_{T} / p_{T}>g_{G} / p_{G}$, and then later, as size increases, $g_{T} / p_{T}<g_{G} / p_{G}$ (see Fig. 5b), favouring initial migration to the tissues, then a switch back to the gut before this would be required for egg release (with a constant ratios this switch should occur at the end of growth when the helminth is of reproductive size).

A second reason why worms may have highly time-dependent increases in mortality rate in the tissues concerns the host response, especially for vertebrates. Thus in the tissues, the mortality rate of the parasite may suddenly rise very steeply due to the host immune response (causing a sudden much steeper increase than that shown in Fig. 5a). A dramatic reduction in mortality might be achieved by migration to the gut, especially at a higher size where the 
parasite is better protected. Such effects would again favour a DTG strategy, with initial growth in the tissues, followed by further growth and finally reproduction in the gut (e.g. Ascaris, etc.).

\subsection{Extension of models to transmission by skin penetration}

Infection by larval penetration of the host's skin is common in trematodes and nematodes. Our models are developed for trophic transmission, but can readily be altered to cover infection by skin penetration. This would require an establishment probability equivalent to $P_{e i}$ or $P_{e j}$, which as before would cancel when considering gut versus tissue exploitation strategies. However, since the worm starts in the tissues, the survivorship term for the migration from the gut to the tissues $M_{G T}$ would be replaced approximately equivalent term $\left(M_{T T}\right)$ to define the survival probability involved in migrating from the skin to the tissue site exploited. If $M_{G T} \cong M_{T T}$, then the mode of transmission (skin penetration versus trophic transmission) does not affect our conditions for the evolution of tissue exploitation in either one-host or two-host cycles.

\section{Acknowledgements}

This work was started with support from NERC (Grant GR3/12774). We thank Manfred Milinski and colleagues at the Max Planck Institute of Evolutionary Biology, Department of Evolutionary Ecology, Plön, for valuable discussions over many years, and two anonymous reviewers for suggestions that have much improved the clarity of the manuscript.

\section{Appendix A. Analysis of nematode life history data in Anderson (2000)}


We classified the range of gut and tissue strategies based on Anderson's (2000) treatise on the life cycles, using the set of definitions shown in Table A1a. For nematode definitive (D) hosts, from Anderson (2000), we discriminated between residence in the host gut (DG; Fig. 1, pathway a) and a variety of tissue migration strategies, usually with return to the gut, ranging from extensive migration from the gut lumen and through the tissues $\left(\mathrm{DT}_{\mathrm{e}} \mathrm{G}\right.$; Fig. 1, pathway c) to minor migrations into the gut mucosa (DT $\mathrm{m}$; Fig. 1, pathway b). Not uncommonly, the nematode larva migrates to a site other than the gut that communicates indirectly or directly with the exterior, and remains there to reproduce $\left(\mathrm{DT}_{\mathrm{e}} \mathrm{O}\right)$. Occasionally, the larva does not show tissue migration, but moves along the gut lumen into some other lumen which connects with the gut, e.g. the lungs or bilary system, in which case we classified the movement as DO, and in very rare instances there was tissue migration followed by egg release into the soma itself $\left(\mathrm{DT}_{\mathrm{eS}}\right)$. For trophic transmission, we excluded the transient phase in the gut after ingestion and before migration, so that if a larva migrates into the tissues soon after infection, and then later returns to the gut for egg release, it would be classified as either $\mathrm{DT}_{\mathrm{m}} \mathrm{G}$ or $\mathrm{DT}_{\mathrm{e}} \mathrm{G}$. In some instances, although egg release in the gut was confirmed, it was not possible to be sure whether there was a tissue migration phase; such data were classified as DG* (for all definitions, see Table A1a).

The information reviewed in Anderson (2000) does not readily permit consistent discrimination between migration strategies in either intermediate (I) or paratenic $(\mathrm{P})$ hosts. We were able to differentiate only between those larvae that remain in the gut of intermediate and paratenic hosts (IG and PG respectively) until transmission to the definitive host, and those that migrate into the tissues and remain in the tissues or body spaces until transmission (IT and PT). We found no evidence for more complex strategies (e.g. ITG) in intermediate or paratenic hosts. For species where the type of host (I or P) can be determined, but no details are recorded in Anderson (2000) for the host site exploited (T or G) we use the categories I? and P?. 
Results are given in Table A1b. For one-host cycles, the single row shows the counts of the number of species having the various strategies in the definitive host. For two-host cycles, row headings are strategies in the intermediate or paratenic host, and column headings are those for the definitive host. Thus the cell in row IT and in the column DG means that the cycle is IT-DG (the larva exploits the tissues of the intermediate host and the gut of the definitive host). For three host life cycles, the table shows only the second intermediate or paratenic host (row) and the definitive host (column). All first hosts for the species with three-host cycles included in Table A1b were intermediates classified as IT (65 cases) or I? (7 cases); two species of Toxocara with three host cycles that had paratenic first hosts were omitted from the analysis.

It seems highly probable that all hosts classified as I? were actually IT, though this could not be verified with certainty from Anderson (2000). We excluded any species that were not internal parasites, or were transmitted by haematophagy, or any species where there was too little information to make a classification. The vast majority (447) species in Table A1b have trophic transmission. We included the few (31) species that have all or part of their transmission by skin penetration, but not those with propagule release through haematophagous invertebrates (nematode Superfamily Filarioidea). For species showing more than one pattern, each was scored as a datum for the respective patterns shown (14\% of the species scored showed more than one pattern).

\section{Appendix B. A second approximation for $w_{1}$}

In our first approximation, the probability of establishment in host $\mathrm{j}, P_{e j}$, was regarded as a constant (see also Parker et al. 2003, Ball et al. 2008, Parker et al. 2009). Realistically, it varies: typically, $P_{e j}=0$ for some time after establishment in the previous host $\mathrm{i}$, then begins to rise at some time $\left(t_{\min }\right)$ before GALM; i.e. $0<t_{\min }<t_{d}$. After GALM (i.e. for $\left.t \geq t_{d}\right), P_{e j}$ 
is typically constant for some long time period. $P_{e j}$ reaches its constant value between $t_{\text {min }}$ and $t_{d}$, and typically just before $t_{d}$; thus $P_{e j}$ can be seen as a small right-angled triangle for $t \leq t_{d}$

Fitness is found by maximising $E=w_{1}+w_{2}$; our second approximation takes $w_{1}$ into account through its dependence on $P_{e j}$.

Ball et al. (2008) have shown that $w_{1}=A \int_{0}^{t_{d}} e^{p_{j} g_{i} t / g_{j}} n d t$ where $A_{G}$ is given by (10). We express $A_{G}$ in terms approximately of the constituent parts of $P_{e j}=0$ for $t<t_{\min }$ $P_{e j}=\left(t-t_{\min }\right) P /\left(t_{d}-t_{\min }\right)$ for $t>t_{\min }$,

where $t_{\min }$ is the time when the parasite first becomes capable of any establishment possibility in $\mathrm{j}$, and $P$ is the value of $P_{e j}$ after GALM.

This is a triangle with area $0.5\left(t_{d}-t_{\min }\right) P$, thus $w_{1}=\left(b W_{j} / p_{j}\right) p_{i j} \int_{0}^{t_{b}} d t e^{-\left(W_{j}-W_{0}\right) / g_{j}+p_{j} g_{i} / / g_{j}}\left(t-t_{\min }\right) P /\left(t_{d}-t_{\min }\right)$.

Unfortunately this integral cannot be evaluated. We try another approximation.

We write

$$
\begin{aligned}
& \int_{t_{\min }}^{t_{d}} d t e^{-\left(W-W_{0}\right) / g_{j}+p_{j} g_{i} t / g_{j}}\left(t-t_{\min }\right) P /\left(t_{d}-t_{\min }\right) \leq \int_{t_{\min }}^{t_{d}} d t e^{-\left(W-W_{0}\right) / g_{j}+p_{j} g_{i} t / g_{j}} P \\
& =P e^{-\left(W-W_{0}\right) / g_{j}+p_{j} g_{j}\left(t_{d}-t_{\min }\right) / g_{j}} \leq P e^{-\left(W-W_{0}\right) / g_{j}} .
\end{aligned}
$$

As an approximation, we look at the minimum and maximum of this amount, take the average, and write $w_{1}=(P / 2) e^{-\left(W_{j}-W_{0}\right) / g_{j}}$.

To determine whether $w_{1}$ used in this approximation behaves differently from the approximation that $w_{1}=0$, we note that our expression for $w_{1}$ is independent of gut or tissue, so the result obtained by putting $w_{1}=0$ holds in this approximation. 


\section{References}

Anderson, R.C., 1992. Nematode Parasites of Vertebrates: their Development and Transmission. first ed. CAB International, Wallingford.

Anderson, R.C., 2000. Nematode Parasites of Vertebrates their Development and Transmission, second ed. CABI Publishing, Wallingford.

Ball, M.A., Parker, G.A., Chubb, J.C., 2008. The evolution of complex life cycles when parasite mortality is size- or time-dependent. J. Theor. Biol. 253, 202-214.

Chubb, J.C., Awachie, J.B.E., Kennedy, C.R., 1964. Evidence for a dynamic equilibrium in the incidence of Cestoda and Acanthocephala in the intestines of freshwater fish. Nature, London 203, 986-987.

Chubb, J.C., Ball, M.A., Parker, G.A., submitted. Living in intermediate hosts: evolutionary adaptations in larval helminths. Trends in Parasitology.

Crompton, D.W.T., 1973. The sites occupied by some parasitic helminths in the alimentary tract of vertebrates. Biol. Rev. 48, 27-83.

Hopkins, C.A., 1959. Seasonal variations in the incidence and development of the cestode Proteocephalus filicollis (Rud. 1810) in Gasterosteus aculeatus (L. 1776). Parasitology $49,529-542$.

Lynch, P.A., Grimm, U., Read, A.F., 2008. How will public and animal health interventions drive life-history evolution in parasitic nematodes? Parasitology 135, 1599-1611.

Iwasa, Y., Wada, G., 2006. Complex life cycle and body sizes at life-history transitions for macroparasites. Evol. Ecol. Res. 8, 1427-1443.

Mulcahy, G., O’Neill, S., Fanning, J., McCarthy, E., Sekiya, M., 2005. Tissue migration by parasitic helminths - an immunoevasive strategy? Trends in Parasitology 21, 273-277.

Parker, G.A., Chubb, J.C., Ball, M.A., Roberts, G.N., 2003. Evolution of complex life cycles in helminth parasites. Nature, London 425, 480-484. 
Parker, G.A., Ball, M.A., Chubb, J.C., 2009. To grow or not to grow? Intermediate and paratenic hosts as helminth life cycle strategies. J. Theor. Biol. 258, 135-147.

Poulin, R., 2007. Evolutionary Ecology of Parasites, second ed. Princeton University Press, Princeton.

Read, A.F., Skorping, A., 1995. The evolution of tissue migration by parasitic nematode larvae. Parasitology 111, 359-371. 
Legends to the figures

Fig. 1. Two-host and one-host trophic life cycles and larval migrations, based on nematodes reproducing in the intestine of definitive hosts. Heavy solid lines outline intermediate and definitive hosts, each with a tubular gut, thin arrowed lines show transmission routes in life cycles. In the two-host life cycle, after propagule ingestion by the intermediate host, larvae are activated to penetrate (bold dashed arrowed line) the gut wall, enter the soma, grow, attain GALM, and become infective. On ingestion of infected intermediate hosts by the definitive host nematode larvae may remain in the intestine (pathway a, intra-intestinal bold arrow), or penetrate the gut wall (solid black lines, pathways $\mathbf{b}, \mathbf{c}$ ). Migration may be limited to the gut mucosa (pathway b) or be extensive within the soma (pathway c). Often the nematodes return to the gut lumen to attain sexual maturity. Some species with one-host life cycles may undergo migrations (pathways b or c, not shown in Figure for one-host life cycles): in some instances (e.g. Ascaris lumbricoides) it is believed that the intermediate host was lost during evolution (Anderson, 2000).

Fig. 2. Evolution of migration from the gut to the tissues. For hosts in which there is growth (a), migration from the gut depends critically on the ratio of growth rate/mortality rate in the two places. The growth rates in the gut and tissues are respectively $g_{G}, g_{T}$, and the total mortality rates in each site respectively $p_{G}, p_{T}$. If the growth/mortality ratio in the tissues $\left(g_{T} / p_{T}\right)$ exceeds that in the gut $\left(g_{G} / p_{G}\right)$, tissue migration can be favoured providing that the costs of migration are sufficiently small (see text). For paratenic hosts (in which there is no growth), the important parameter is simply the mortality rates, $p_{G}, p_{T}$ (see text).

Fig. 3. Threshold lines for selection to favour DTG or DG in a one-host cycle, iterated from condition (4b). Each line plots the threshold $W_{T}^{*}$ as a function of $W_{G}^{*}$; above each line DTG is 
favoured, below each line, DG is favoured (see text). The three threshold lines are for different values of $M_{G T j} M_{T G j}$ (shown on each line). $W_{0}=1$.

Fig. 4. Threshold lines for selection to favour IT-DG or IG-DG in a two-host cycle, iterated from condition (12c). Curves are the threshold value for $M_{G T i}$, the probability of surviving tissue migration, plotted against the total growth up to GALM in host i $\left(W_{d}-W_{0}\right)$. Strategy IT-DG is favoured above each line, and IG-DG is favoured below each line. For all curves, transmission rates were assumed to be equal for gut and tissues; i.e. $p_{G i j}=p_{T i j}=0.02$. For the continuous curves mortality rates did not change at GALM. For the upper continuous curves, mortality rates were equal in the gut and tissues, i.e. $p_{G i}^{1}=p_{G i}^{2}=p_{T i}^{1}=p_{T i}^{2}=0.02$; for the lower continuous curves, those in the tissues were half those in the gut, i.e. $p_{G i}^{1}=p_{G i}^{2}=0.02$, $p_{T i}^{1}=p_{T i}^{2}=0.01$. In the lower broken curves, both mortality rates were reduced to half their former value at GALM, i.e. $p_{G i}^{1}=0.02, p_{G i}^{2}=0.01, p_{T i}^{1}=0.01, p_{T i}^{2}=0.005$. In the lower dotted curves, at GALM only the mortality rate in the tissues was reduced to half its former value at GALM, i.e. $p_{G i}^{1}=0.02, p_{G i}^{2}=0.02, p_{T i}^{1}=0.01, p_{T i}^{2}=0.005$. In (a) growth rate in the tissues was twice that in the gut, i.e. $g_{T i}=0.2, g_{G i}=0.1$; in (b) growth rate in the tissues was only 20 per cent greater than that in the gut, i.e. $g_{T i}=0.12, g_{G i}=0.1$.

Fig. 5. Size- or time-dependent changes in mortality rate causing a switch in the growth rate/mortality rate asymmetry between the gut (G; continuous line) and the tissues ( $\mathrm{T}$; broken line) during growth in a definitive host $\mathrm{j}$. (a) The noise mortality in host $\mathrm{j}$ may be higher in the gut for small larvae, but as the larva grows it becomes less vulnerable to peristaltic and other mechanical processes, reducing the larval mortality rate. In contrast, in the tissues, larval mortality may be initially lower, but may rise as larval size increases due to increased costs imposed on the host, or because the host response increases. (b) The growth rate/mortality rate ratio in the gut and tissues shows the reverse trend, making the tissues 
initially favourable $\left(g_{T j} / p_{T j}>g_{G j} / p_{G j}\right)$, but at some time after the switch line when $g_{G j} / p_{G j}>$ $g_{T j} / p_{T j}$, migration to the gut can be favoured for continued growth. 
Table 1. Strategies for theoretical analysis after a larva infects the final (definitive) host. For two host cycles, prefixes for intermediate hosts, IG and IT, are replaced for paratenic hosts with PG and PT; growth occurs only in I hosts and not in P hosts.

\section{One host cycles}
DG Growth, sexual maturation, and release of propagules occurs in the host gut
DT Growth occurs in the host tissues; after sexual maturation, propagules are released away from the gut
DTG All growth occurs in the tissues, followed by movement to the gut for release of propagules
DGT All growth occurs in the gut, followed by movement to the tissues and release of propagules away from the gut

\section{Two host cycles}

IG-DG

The parasite exploits the gut of the intermediate host, and the gut of the definitive host

IT-DG The parasite exploits the tissues of the intermediate host, and the gut of the definitive host

IT-DT

The parasite exploits the tissues of the intermediate host, and the tissues of the definitive host

IG-DT The parasite exploits the gut of the intermediate host, and the tissues of the definitive host

The parasite exploits the gut of the intermediate host, and in the

IG-DGT definitive host completes growth in the gut and then migrates to the tissues for reproduction

The parasite exploits the tissues of the intermediate host, and in the

IT-DTG definitive host migrates to the tissues for growth and returns to the gut for egg release 
Table 2. Relative merits for various life history parameters of host gut and tissue sites for exploitation by helminths (see text).

Life

history paramete

$\mathbf{r}$

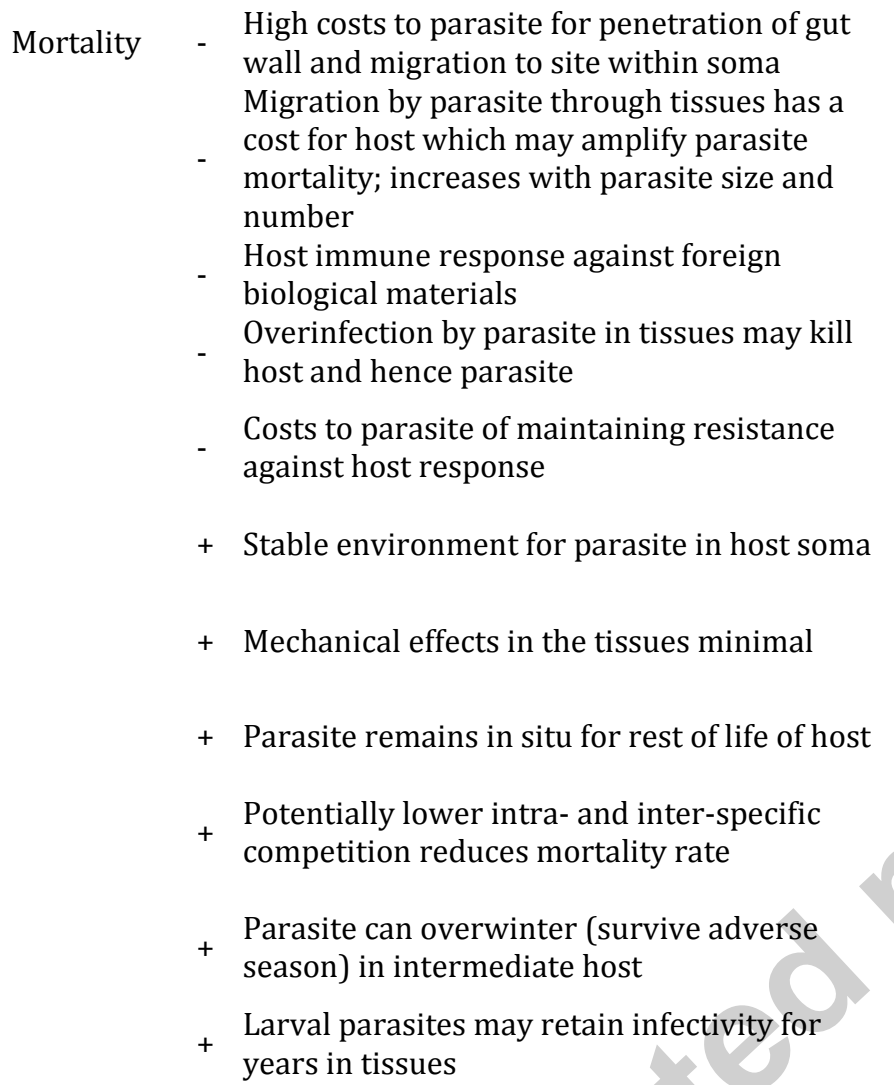

Trans- $\quad+$ Predators that consume only parts of prey mission ${ }^{+}$may have preferences for the tissues

Constant, potentially high quality nutrient

Growth + resources in host tissues enhances growth rate of parasite

If parasite growth rate high and parasite remains in the same tissue site, may be drain on local host tissue nutrient resources limiting growth rate

Reproduction

\section{- Cross-fertilization opportunities reduced}

Propagule release only through death of host

- unless helminth located by a lumen with access to the exterior

Unless helminth located by a lumen with access to the exterior, release by death of host a one-off event minimising dissemination of parasite

\section{Host gut}

+ Low costs to parasite for movement to specific niche in gut

+ No migration therefore no cost for host tissue damage

+ No host immune response against biological materials in gut lumen

+ Overinfection by parasite in gut lumen unlikely to kill host Costs to parasite of maintaining resistance

+ against host response only if it browses on host mucosa

Unstable environment for parasite in gut lumen through peristalsis

Mechanical effects of raw (non-digested) food

- may affect survival of parasite in anterior regions of gut

Parasite constantly needs to maintain position in gut lumen to avoid expulsion

Potentially higher intra- and inter-specific competition in gut lumen increases mortality rate Parasite in gut lumen can be lost when host hibernates overwinter (or through adverse season)

Relatively few gut-inhabiting adult parasites survive for more than a year

Predators that consume only parts of prey may avoid eating the gut

Potentially lower quality nutrient resources (semi-digested host food) in gut lumen change with host feeding patterns to affect growth rate of parasite

+ Host feeding activity regularly replaces nutrients in gut lumen

+ Cross-fertilization opportunities enhanced

+ Propagule release to exterior continuous with the host faeces

Host movement and defaecation ensures

+ continuous and wide dissemination of parasite propagules 
Unless propagule release occurs through haematophagous invertebrates (nematode

- Superfamily Filarioidea), less protection and attraction of next host for dissemination of parasite
Host faeces protect parasite propagules and + attract potential hosts to enhance dissemination of parasite 
Table A1a. Definitions of nematode life history strategies used for our analysis of Anderson (2000).

\section{Strategies in definitive host}

DG

After ingestion of parasite, growth, sexual maturation and propagule release all occur in the lumen of the host gut

After ingestion of parasite there is immediate migration to the host gut mucosal tissue or its

$\mathrm{DT}_{\mathrm{m}} \mathrm{G}$ associated glands, followed by growth, return to the gut with propagule release into the lumen (or between mucosa and koilin layer)

After ingestion of parasite there is immediate extensive migration through the host tissues

$\mathrm{DT}_{\mathrm{e}} \mathrm{G}$ followed by growth, return to the gut with propagule release into the gut lumen (or between mucosa and koilin layer)

Propagules are released into the definitive host gut lumen (or between mucosa and koilin layer),

$\mathrm{DG}^{*} \quad$ but unclear whether there is tissue migration in definitive host (i.e. may be $\mathrm{DG}$ or $\mathrm{DT}_{\mathrm{m}} \mathrm{G}, \mathrm{DT} \mathrm{e}$ )

After ingestion of parasite, there is no tissue migration but immediate movement along the gut and its associated ducts to a site where growth and sexual maturation occur; propagules are

DO released into some lumen other than the gut that opens either directly (e.g. urino-genital system) or indirectly via the gut (e.g. nasal cavities, lungs, bilary system, pancreatic system, swim bladder) to the exterior

After ingestion of the parasite there is immediate migration through the host tissues followed by growth and eventual propagule release either direct to the exterior, or into some lumen other

$\mathrm{DT}_{\mathrm{e}}$ than the gut that opens either directly (e.g. urino-genital system) or indirectly via the gut (e.g. nasal cavities, lungs, bilary system, pancreatic system, swim bladder) to the exterior

Propagules are released into some lumen other than the gut that opens either directly (e.g.

DO* urino-genital system) or indirectly via the gut (e.g. nasal cavities, lungs, bilary system, pancreatic system, swim bladder) to the exterior, but unclear whether there is larval tissue migration in definitive host (i.e. may be $\mathrm{DO}$ or $\mathrm{DT}_{\mathrm{e} 0}$ )

After ingestion of the parasite there is immediate migration through the host tissues where

$\mathrm{DT}_{\mathrm{eS}}$ growth and propagule release occurs, but eggs are released into some part of the soma having no direct communication with the exterior (e.g. blood system or somatic parenchyma)

After ingestion of parasite there is a growth phase in the lumen of the host gut, followed by

DGT migration through the host tissues to release propagules in any site included in definitions $\mathrm{DT}_{\mathrm{e}}$, $\mathrm{DT}_{\mathrm{eS}}$ )

\section{Strategies in intermediate or paratenic host}

Propagule ingested by intermediate host, larval growth in lumen of gut where the larva remains until eaten by the next host

Propagule ingested by the paratenic host where it remains without growth in lumen of gut until eaten by the next host

After the propagule is ingested by intermediate host there is immediate migration to the host tissues or body spaces where the larva grows to GALM and remains until eaten by the next host

After the propagule or larva is ingested by paratenic host there is immediate migration to the host tissues or body spaces where it remains without growth until eaten by the next host

It is known that there is an intermediate or paratenic host, but no details recorded in Anderson

I?, P? (2000) for host site exploited 
Table A1b. Number of species of nematodes from Anderson (2000) showing each life history pattern as defined in Table 1a.

\begin{tabular}{|c|c|c|c|c|c|c|c|c|c|c|c|}
\hline & & DG & $\mathrm{DT}_{\mathrm{m}} \mathrm{G}$ & $\mathbf{D T}_{\mathrm{e}} \mathrm{G}$ & DG* & DO & $\mathbf{D T}_{\mathrm{eo}}$ & DO* & $\mathrm{DT}_{\mathrm{eS}}$ & DGT & Tota \\
\hline \multirow[t]{5}{*}{ One host cycles } & & 23 & 57 & 51 & 20 & 0 & 13 & 2 & 1 & 0 & \\
\hline & & \multicolumn{4}{|c|}{$\begin{array}{l}\text { total releasing propagules in } \\
\qquad \text { gut }=151\end{array}$} & \multicolumn{5}{|c|}{$\begin{array}{l}\text { total releasing propagules away } \\
\text { from gut }=16\end{array}$} & 167 \\
\hline & IG & 0 & 0 & 0 & 0 & 0 & 0 & 0 & 0 & 0 & 0 \\
\hline & IT & 65 & 51 & 20 & 41 & 3 & 30 & 3 & 8 & 0 & 221 \\
\hline & I? & 0 & 3 & 0 & 5 & 0 & 25 & 0 & 9 & 0 & 42 \\
\hline \multirow[t]{7}{*}{ Two host cycles } & & \multicolumn{4}{|c|}{$\begin{array}{l}\text { total releasing propagules in } \\
\text { gut }=185\end{array}$} & \multicolumn{5}{|c|}{$\begin{array}{l}\text { total releasing propagules away } \\
\text { from gut }=78\end{array}$} & 26 \\
\hline & PG & 1 & 1 & 0 & 0 & 0 & 0 & 0 & 0 & 0 & 2 \\
\hline & PT & 0 & 1 & 2 & 1 & 0 & 4 & 0 & 0 & 0 & 8 \\
\hline & P? & 0 & 1 & 1 & 1 & 0 & 4 & 0 & 0 & 0 & 7 \\
\hline & & \multicolumn{4}{|c|}{$\begin{array}{l}\text { total releasing propagules in } \\
\text { gut }=9\end{array}$} & \multicolumn{5}{|c|}{$\begin{array}{l}\text { total releasing propagules away } \\
\text { from gut }=8\end{array}$} & 17 \\
\hline & IG & 0 & 0 & 0 & 0 & 0 & 0 & 0 & 0 & 0 & 0 \\
\hline & IT & 9 & 6 & 2 & 1 & 0 & 0 & 0 & 0 & 0 & 18 \\
\hline \multirow{5}{*}{$\begin{array}{l}\text { Three host } \\
\text { cycles (data for } \\
\text { last two hosts; } \\
\text { first host is IT } \\
\text { or I?) }\end{array}$} & & \multicolumn{4}{|c|}{$\begin{array}{l}\text { total releasing propagules in } \\
\qquad \text { gut }=18\end{array}$} & \multicolumn{5}{|c|}{$\begin{array}{l}\text { total releasing propagules away } \\
\text { from gut }=0\end{array}$} & \\
\hline & PG & 4 & 0 & 0 & 1 & 0 & 0 & 0 & 1 & 0 & 6 \\
\hline & PT & 6 & 10 & & 6 & 0 & 12 & 1 & 2 & 0 & 40 \\
\hline & P? & 2 & 3 & 0 & 2 & 0 & 1 & 0 & 0 & 0 & 8 \\
\hline & & \multicolumn{4}{|c|}{$\begin{array}{l}\text { total releasing propagules in } \\
\text { gut }=37\end{array}$} & \multicolumn{5}{|c|}{$\begin{array}{l}\text { total releasing propagules away } \\
\text { from gut }=16\end{array}$} & \\
\hline
\end{tabular}

